\title{
ERRATA
}

Bacteroides intermedius comb. nov. and Descriptions of Bacteroides corporis sp. nov. and Bacteroides levii sp. nov. JOHN L. JOHNSON AND LILLIAN V. HOLDEMAN

Department of Anaerobic Microbiology, College of Agriculture and Life Sciences, Virginia Polytechnic Institute and State University, Blacksburg, Virginia 24061

Volume 33, no. 1, p. 21, Table 6: The indole reaction for Bacteroides loescheii should be "-," not "+."

\section{Relationships Among Catalase-Positive Campylobacters Determined by Deoxyribonucleic Acid-Deoxyribonucleic Acid Hybridization}

SYDNEY M. HARVEY AND JAMES R. GREENWOOD

Irvine Diagnostic Services, Irvine, California 92175, and Public Health Laboratory, County of Orange, Santa Ana, California 92702

Volume 33, no. 2, p. 281 , column 1, lines 9 through 12 : Should read " . . as a $C$. jejuni biotype 2 strain. Strain C-85 was from a symptomatic patient and was classified by us as a $C$. jejuni biotype 1 strain by both. ..."

Lactobacillus yamanashiensis subsp. yamanashiensis and Lactobacillus yamanashiensis subsp. mali sp. and subsp. nov., nom. rev.

H. NONOMURA

Faculty of Engineering, Yamanashi University, Takeda 4, Kofu, Japan

Volume 33, no. 2, p. 406, column 2, line 27: "ATCC 27340” should read "ATCC 27304." 